\title{
Germanica
}

GERMANICA

$58 \mid 2016$

Le roman policier dans l'espace germanophone

\section{„das, was wir beschreiben, ist immer noch weniger schlimm als oft die Realität" - der aufstörende Charakter von Sebastian Fitzeks und Michael Tsokos' Roman Abgeschnitten}

"Ce que nous décrivons est toujours moins grave que ne l'est souvent la réalité" le caractère irritant du roman de Sebastian Fitzek et Michael Tsokos

Abgeschnitten

"that what we are going to describe frequently is less tremendously than the reality." The novel Abgeschnitten by Sebastian Fitzek and Michael Tsokos

Monika Wolting

\section{(2) OpenEdition}

Journals

Édition électronique

URL : http://journals.openedition.org/germanica/3221

DOI : 10.4000/germanica.3221

ISSN : 2107-0784

Éditeur

Université de Lille

Édition imprimée

Date de publication : 30 septembre 2016

Pagination : 211-225

ISBN : 9782913857377

ISSN : 0984-2632

Référence électronique

Monika Wolting, " „das, was wir beschreiben, ist immer noch weniger schlimm als oft die Realität" der aufstörende Charakter von Sebastian Fitzeks und Michael Tsokos' Roman Abgeschnitten », Germanica [Online], 58 | 2016, Online erschienen am: 30 September 2018, abgerufen am 06 Oktober 2020. URL : http://journals.openedition.org/germanica/3221 ; DOI : https://doi.org/10.4000/ germanica.3221 


\title{
„das, was wir beschreiben, ist immer noch weniger schlimm als oft die Realität" - der aufstörende Charakter von Sebastian Fitzeks und Michael Tsokos' Roman Abgeschnitten
}

\author{
Monika WOLTING \\ Universität Wrocław
}

\section{Aufstörung durch Text}

Es sei einleitend mit einer Überlegung des Rechtsmediziners Michael Tsokos aus dem Jahre 2012 begonnen:

Ich finde, dass es eine intensive öffentliche Auseinandersetzung zu dem Thema [härtere Strafen für Gewalttäter und Sexualverbrecher] geben sollte: dass wir darüber diskutieren, was es bedeutet und was es für die Rechtsprechung bedeuten sollte, wenn ein Kind missbraucht, wenn ihm möglicherweise seine Kindheit genommen wird und ihm schwere psychische Schäden zugefügt werden; oder wenn jemand in einer U-Bahn-Station so zusammengeschlagen wird, dass er den Rest seines Lebens in einem Rollstuhl verbringen muss ${ }^{1}$.

1. - Sabine Schmidt, „Wo ist hier bitte die Minzpaste? “ Interview mit Sebastian Fitzek und Michael Tsokos. 22. Oktober 2012. In: http://www.buchjournal.de/554398/ 
Tsokos macht hier auf ein Problem aufmerksam, das in der Gesellschaft eine scheinbar offene Diskussion benötigt. Er plädiert für eine breit angelegte Aufnahme eines für die Gesellschaft relevanten Themas: der härteren und konsequenteren Bestrafung von Gewalttätern, denn seiner Auffassung nach vernachlässigt der Staat seine Pflichten den Opfern von Gewaltdelikten gegenüber. Dies belegt er in seiner 2014 erschienen Streitschrift Deutschland misshandelt seine Kinder ${ }^{2}$, die zu einer Irritation in verschiedenen Gremien, wie dem Deutschen Richterbund, dem Verband Deutscher Kinder- und Jugendärzte, dem Deutschen Kinderschutzbund geführt hatte ${ }^{3}$. Zu den Gründen für das Entstehen des umstrittenen Bandes äußert sich die Co-Autorin Saskia Guddat:

Wir haben uns dafür bewusst entschieden, eine Streitschrift zu schreiben, denn wenn wir ein weiteres wissenschaftliches Buch geschrieben hätten, säßen wir nicht hier [in der Sendung von Markus Lanz, ZDF], dann hätten es auch nicht so viele Leute gelesen, denn die wissenschaftlichen Bücher gibt es bereits. Ich glaube, dass viele sich an der Streitschrift gestoßen haben und die Dinge persönlich genommen haben, anstatt zu sehen, wie wir es formuliert haben. Wir müssen das Thema der Kindesmisshandlung in den Fokus stellen, wir müssen uns als Gesellschaft damit auseinandersetzen, um dann zu schauen, was sind die Schwachstellen des Systems, das wir haben ${ }^{4}$.

An Guddats Äußerung sind zwei wichtige Merkmale zu beobachten, die systemtheoretisch diskutiert werden können. Erstens: Eine Streitschrift hat im Gegensatz zu einem Sachbuch die Fähigkeit, das öffentliche Interesse zu wecken. Zweitens: Erst dies führt zur Auseinandersetzungen mit einem nonkonformen Thema. Die Autoren wählten ein Genre, das ihnen versprach, ein größeres Publikum zu erreichen und ihr Anliegen öffentlich zu machen, denn die Ausführungen zum behandelten Thema, die in wissenschaftlichen Abhandlungen getätigt wurden, zeigten offenbar keine Wirkung. Erst das Einschalten großer Teile der Gesellschaft führte zum Anstoß zu einer bundesweiten Debatte, um die es den Autoren ging. Unter Bezug auf Luhmann kann festgestellt werden, dass „Umweltphänomene“ dann aufgestört werden, wenn sie einen Informationsverarbeitungsprozess in Gang setzten, mithin eine auf (Re)Stabilisierung angelegte innersystemische Kommunikation anregen. Genau das beabsichtigen die Autoren der Streitschrift: Ein etabliertes System durch ihr Schreiben und durch das

2. - Michael Tsokos, Saskia Guddat, Deutschland misshandelt seine Kinder, München, Droemer, 2014.

3. - https://www.youtube.com/watch?v=eOoHKVExekQ.

4. - https://www.youtube.com/watch?v=eOoHKVExekQ. 
Einschalten einer breiteren Öffentlichkeit aufzustören, um auf gravierende Verstöße aufmerksam zu machen und womöglich, wenn der Druck des Systems der Öffentlichkeit auf das System der Rechtsprechung in Deutschland stark genug wird, in dem zweiten zu Veränderungen zu führen.

Das Anliegen dieses Beitrags ist es, den möglichen aufstörenden Charakters nicht einer Streitschrift, sondern eines literarischen Textes aufzuzeigen. Es wird davon ausgegangen, dass Aufstörungen im System Literatur schwieriger zu erzeugen sind als im Mediensystem. Denn anders als etwa in diesen gesellschaftlichen Teilsystemen, gehört das Durchspielen von Störungen zu den Eigenschaften des „Systems Literatur". So erscheint Literatur als der Raum, in dem Störungen schon immer symbolisch ausgehandelt werden könnten. Man kann es auch mit Niklas Luhmann definieren: Mit Kunst und Literatur schaffen Gesellschaftssysteme sich Formen der Autopoiesis, ,um sich selbst zu beobachten: in sich selbst gegen sich selbst ${ }^{5}$."

\section{Das „Was“ und das „Wie“ des Erzählens}

2012 schrieb Michael Tsokos gemeinsam mit dem Krimiautor Sebastian Fitzek einen Thriller unter dem Titel Abgeschnitten.

Rechtsmediziner Prof. Dr. Paul Herzfeld findet im Kopf einer durch menschliches Einwirken entstellten Leiche eine Kapsel mit der Telefonnummer seiner eigenen Tochter. Herzfeld lebt von seiner Frau getrennt und hat inzwischen den Kontakt zu seiner Tochter fast ganz verloren. Hannah wurde entführt, das erfährt Herzfeld aus einer Mailboxansage. Der psychopathische Entführer hat eine weitere Leiche auf Helgoland mit Hinweisen über den Verbleib von Hannah präpariert. Herzfeld hat jedoch keine Chance, selbst an die Informationen zu kommen. Die Hochseeinsel ist durch einen Orkan vom Festland abgeschnitten, die Bevölkerung zum größten Teil bereits evakuiert. Unter den wenigen Menschen, die sich noch auf der Insel aufhalten, befindet sich die Comiczeichnerin Linda, die den Toten am Strand gefunden hat. Linda versteckt sich auf der Insel, denn sie wird von einem Stalker bedroht. Herzfeld versucht Linda dazu zu überreden, die Obduktion der gefundenen Leiche nach seinen telefonischen Anweisungen durchzuführen. Linda willigt ein. Die Situation spitzt sich zu, eine weitere Tote wird auf der Insel von Linda und ihrem Helfer Elder vorgefunden und der Stalker deutet seine Anwesenheit in der Pathologie des durch den Sturm stillgelegten Krankenhauses durch ein mehrmaliges verstecktes Erscheinen an. Im weiteren Verlauf der Geschichte verbindet Herzfeld

5. - Niklas Luhmann, Einführung in die Systemtheorie, Heidelberg, Carl-AuerVerlag, 2006. 
alle Opfer und Täter zu einer Geschichte, es handelt sich dabei um einen Racheakt, der an ihm ausgeübt werden soll. Vor einiger Zeit wurde die Tochter des Kollegen von Herzfeld, Martinek, Opfer eines Sexualwiederholungstäters. Herzfeld führte die Obduktion an dem toten Mädchen durch und stellte Suizid und keinen Mord als Todesursache fest. Martinek bat ihn, das Gutachten zu fälschen, um den Täter wegen Mordes und nicht wegen Entführung und Vergewaltigung verurteilen zu lassen. Herzfeld weigerte sich, falsche Daten in seinen Obduktionsbericht zu schreiben. Der Täter Sadler wurde zu einer Haftstrafe wegen fahrlässigen Totschlags verurteilt, wegen guter Führung kam er vorzeitig nach knapp drei Jahren aus dem Gefängnis. Einige Tage nach der Entlassung entführte er ein weiteres Mädchen, Rebecca Schwintowski, das ebenfalls unter dem enormen Druck der Misshandlungen das Leben nahm. Schwintowski und Martinek beschlossen im Zuge der Selbstjustiz, die Täter (den Vergewaltiger und die Richterin, die nach der Einschätzung der beiden Väter eine zu milde Strafe verhängt hatte) zu bestrafen und dem Rechtsmediziner und dem ganzen System einen Denkzettel zu verpassen:

Im ersten Impuls wollte ich [Schwintowski] Sadler zu Tode foltern und die Richterin von meinen Leuten erledigen lassen. Aber dann machte Martinek mir klar, dass es hier nicht nur um uns geht. [...] Es geht um das gesamte System, dass Opfer zu Tätern macht ${ }^{6}$.

Wenn man nach dem „Wie“ des Erzählens fragt, wird offenbar, dass der Text durch den Wechsel des point of view bestimmt ist. Die Geschichte wird von einem heterodiegetischen Erzähler erzählt. Der Aufbau der nacheinander folgenden Episoden ist meistens linear bis synchron. Der Erzähler befindet sich gleichzeitig an zwei Orten: auf dem Festland hier begleitet er mit seiner Erzählung das Geschehen um Paul Herzfeld und auf der Hochseeinsel Helgoland, wo er von den Geschehnissen um die Comiczeichnerin Linda berichtet. Die Gegenwartsebene wird durch eine, ebenso in kurzen Kapiteln erzählte Episode „In der Höhle“ unterbrochen, die die meisten Gewaltszenen enthält. Allerdings wird erst zum Textende augenfällig, dass es sich hierbei um eine Analepse, also um eine Erzählung auf der Vergangenheitsebene, handelt. Diese Technik führt zur Spannungssteigerung des Thrillers.

In die den Text überwiegend bestimmende Erzählerrede mischt sich häufig Figurenrede:

Auf der einen Seite schien es keinen Zweifel daran zu geben, dass Martinek hinter all dem Schrecken steckte. Sein ehemaliger Kollege

6. - Sebastian Fitzek/ Michael Tsokos, Abgeschnitten, München, Droemer, 2012, S. 294f. Im Folgenden mit dem Kürzel A ausgewiesen und mit Seitenzahl versehen. 
hatte die Hauptschuldigen für den Tod seiner eigenen Tochter büßen lassen, allen voran Jan Erik Sadler. [ ] Danach hatte die Richterin dran glauben müssen.

Und jetzt bin ich an der Reihe.

Das Auto wurde von einer Schneewehe getroffen, und Herzfeld bremste instinktiv ab.

Jetzt soll ich erfahren, was es heißt, die einzige Tochter zu verlieren, weil ich dir damals nicht geholfen habe, die Beweise zu fälschen.

Vieles sprach dafür, dass Hannah nicht mehr am Leben war (A, 243). [Markierungen im Text vorhanden]

In dieser Episode wechselt der Point of view mehrmals, die Erzählerrede wird von der Figurenrede unterbrochen. Die Figuren treten kurz als Ich-Erzähler immer wieder auf und erzählen direkt von ihren Befürchtungen, Gedanken, Emotionen. Diese Textstellen sind von der Wiedergabe der Figurenrede, die in Formen von wiedergegebenen Dialogen, die mit Anführungszeichen markiert sind, zu unterscheiden. Dieser Wechsel hat zum einen die Funktion der Beschleunigung des Erzählfortgangs, zum anderen aber auch die genauere Charakterisierung des Figurenverhaltens. Zudem bringt die Figurenrede dem Leser die Figuren näher, der Leser ist dann eher bereit, Empathie zu entwickeln, einen Blickwechsel vorzunehmen, die Sichtweise der dargestellten Figur zu übernehmen. Erst in den Darbietungen der Gedanken der Figuren wird die Grausamkeit der dargestellten Situationen ersichtlich. Die unmittelbare Konfrontation mit den Ängsten der Figuren lässt deren psychische Belastung erahnen und ihre Ausweglosigkeit spüren. In Äußerungen, wie:

Das Handtuch, dessen Berührung ein Gefühl vollkommenen Ekels ausgelöst hatte. Denn es war nass. Jemand musste sich damit abgetrocknet haben, während sie unter der Dusche gestanden hatte (A, 21). Das Bett ist warm. Der Geruch noch intensiv. Er ist immer noch im Haus (A, 28-29).

spricht die Betroffene selbst, sie übernimmt kurz die Rolle eines homodiegetischen Erzählers, so könnte man vermuten, dass hier ein unglaubwürdiger Erzähler kurz in Erscheinung tritt. Es können also auch paranoide Störungen der Figur sein, die keine wahrheitsgetreue Entsprechung haben. Der Erzähler ermöglicht den Figuren selber zu sprechen, denn gerade ihre Äußerungen sind es, die aufstören. Die Vermutungen und damit verbundenen Ängste Lindas wurden von der Polizei als ,paranoide Störungen“ wahrgenommen und ihr Fall als unsinnig und überspannt behandelt. Der Leser kann aber durch sein Einfühlungsvermögen und die Fähigkeit zur Perspektivenübernahme, die durch das direkte Einsetzen der Figurenperspektive angeregt 
werden, Lindas Befürchtungen Glauben schenken und so das Ausmaß ihres Traumas begreifen.

\section{Gesellschaftsrelevante Themen}

Fitzek und Tsokos haben einen Thriller geschrieben, der sich durch einen starken Plot, gut aufgebaute Spannungsbögen, in sich stimmige Figuren und ansprechende Settings charakterisiert. Das Besondere an diesem Text ist allerdings nicht die spannende Geschichte, sondern die Anbindung des Plots an gesellschaftsrelevante Themen. Dazu gehört das Thema Stalking (mit der Einführung der Figuren Danny und Linda), Sexualmissbrauch von Jugendlichen (die Figur Rebecca, die in ihrem Verlies unbeschreibliche Qualen und Folter erleben muss), erneute Straffälligkeit nach früherer Bestrafung (am Beispiel von Jan Erik Sadler, der einige Tage nach der Entlassung erneut ein Mädchen verschleppt und vergewaltigt), Selbstjustiz (am Beispiel der Figuren Martinek, Schwintowski und Herzfeld, die keine andere Möglichkeit sehen, Gerechtigkeit widerfahren zu lassen, als selbst zu morden) und nicht zuletzt der Schwerpunkt des Textes, der sich auf alle vorigen Phänomene bezieht: das Versagen des Rechtssystems im Strafverfahren.

Carsten Gansel schreibt, dass sich „Gesellschaften in Form von Kunst, Musik, Literatur mediatisierte Strukturen leisten, deren Störcharakter toleriert, in unterschiedlichem Maße kontrolliert und in differenter Skalierung erwünscht ist ${ }^{7}$." Wenn man Literatur als Modell der Wirklichkeit fasst, das gegebene gesellschaftliche Zustände und Prozesse - die „Präfiguration“ eines Stoffes im Sinne Ricoeurs - in literarische Formen umwandelt und damit für die Leser erfahrbar macht, dann ließe sich der Roman als ein Versuch lesen, die Gesellschaft durch das Aufzeigen des Leidens der Opfer des Strafsystems aufzustören. Es zeigt die Notwendigkeit, das gegebene Strafsystem zu verändern, seine Zuständigkeitsbereiche für Täter und Opfer neu zu formulieren, neue Akzente in der Strafordnung, der psychologischen Betreuung, sowohl der Täter als auch der Opfer zu setzen. Dieser Text offenbart in aller Deutlichkeit, wo und wie das deutsche Rechtssystem im Strafverfahren versagt.

\section{Aufstörung durch ,Abgeschnitten“}

Wollte man nunmehr konkret Störungen im Bereich Literatur erfassen, erweist es sich als produktiv, zwischen Handlungs- bzw.

7. - Carsten Gansel, ,Zu Aspekten einer Bestimmung der Kategorie 'Störung'Möglichkeiten der Anwendung für Analysen des Handlungs- und Symbolsystems Literatur“', in: Carsten Gansel, Norman Ächtler, Das 'Prinzip Störung' in den Geistesund Sozialwissenschaften, Berlin, De Gruyter, 2013, S. 36. 
Sozialsystem auf der einen und Symbolsystem Literatur auf der anderen Seite zu unterscheiden ${ }^{8}$. Im literarischen Handlungssystem kann es zu Störungen auf den Ebenen von Produktion, Distribution und Rezeption kommen. Im Symbolsystem, also in den aus den Handlungsrollen von Produktion und Distribution hervorgegangenen Texten mit ihren Stoffen, Themen, Darstellungsweisen, betreffen Störungen das „Was“und „Wie“ der Darstellung. Bekannt ist, dass Kriminalromane stets Grenzen der Moral, der Ästhetik überschreiten. Der Verstoß gegen die zugelassenen gesellschaftlichen Normen liegt in der Art der Gattung und führt selten zu Aufstörungen im gesamtgesellschaftlichen System. Filme mit besonderem Gewaltpotenzial werden mit einer Altersgrenze versehen, im Falle literarischer Texte sind die Verlage nicht verpflichtet, Informationen diesbezüglich zu liefern. Fitzek und Tsokos übertreten aber mit der Wahl des Themas und der Auslegung einiger Gedanken sehr wohl den für einen Krimi zugeschnittenen Toleranzraum. Denn einer der Autoren gehört nicht dem Literatursystem, sondern dem Rechtssystem an. Damit gewinnen die Handlungen, die Figuren und die im Text getätigten Aussagen eine gesteigerte Realitätsnähe. Wenn zu der Analyse auch die Paratexte, die den Anfang des Romans bilden herangezogen werden:

Das Landgericht hatte den 61-jährigen Mann, wie berichtet, zu zwei Jahren Haft auf Bewährung verurteilt, nachdem er sexuellen Missbrauch seiner Tochter in 282 Fällen gestanden hatte. Der Täter profitierte davon, dass viele Jahre vergingen, ehe das Opfer über das Erlebte sprechen konnte. Quelle: Der Tagesspiegel vom 16.April 2010 (A, 5).

Das Landgericht Hamburg hat den Börsenbetrüger Rüdiger Beuttenmüller zu einer Freiheitsstrafe von fünfeinhalb Jahren verurteilt. Der Geschäftsmann hatte Millionen von Billigaktien gekauft, danach deren Kurse durch Falschinformationen in die Höhe getrieben - und dann die Anteile verkauft, bevor deren Preise wieder abstürzte. Quelle: Frankfurter Allgemeine Zeitung vom 17. April 2009 (A, 5).

dann wird der Realitätsbezug nicht nur auf der Handlungsebene, sondern auch auf der Handlungsmusterebene noch deutlicher. Damit übernimmt der Roman im Handlungssystem Literatur eine weitere Rolle als nur spannende Handlung und Rätsel zu liefern. Die Inhalte und die Figurenanlage weisen auf gewichtige Problemfelder in der deutschen Gesellschaft hin und wollen damit die Gesellschaft aufstören, daher die aussagekräftige Zusammenstellung der Paratexte.

Bei der Frage nach Störungen in „Abgeschnitten“ ist es wichtig, den Blick zunächst auf das Symbolsystem zu richten, also auf den Text selbst. Denn: Es ist das „Was und Wie“ des Erzählens, das bishe-

$$
\text { 8. - Ebd. }
$$


rige Erwartungen, Normen, Werte, Konventionen, die innerhalb des Teilsystems des Kriminalromans gelten, irritiert und aufstört und gegebenenfalls gesetzte Grenzen des Systems überschreitet. Erst wenn ein Text diese Voraussetzungen erfüllt, besteht die Chance, dass ein Autor und sein Text dann auch im Handlungssystem als Störung wahrgenommen werden. Im Falle von Tsokos und Fitzek kann man von einer Präsenz der Autoren in der Öffentlichkeit sprechen, die eine Diskussion in der Gesellschaft in Gang gesetzt hat. Dass dies erfolgt ist, zeigen die vielen Blog- Eintragungen, die zahlreich geführten Gespräche mit den beiden Autoren, in denen sie des Öfteren den Realitätsbezug des fiktiven Textes betonen:

Buchjournal: Warum war es Ihnen wichtig, über den Umgang mit Sexualstraftätern zu schreiben?

Sebastian Fitzek: Wir hatten uns das nicht vorgenommen, das Thema hat sich erst beim Schreiben ergeben. Es hat mich immer wieder geärgert und musste dann offensichtlich verarbeitet werden, wenn ich in einer Zeitung gelesen habe, dass ein Sexual- oder auch ein anderer Intensivstraftäter mit nur einer sehr geringen Haft- oder einer Bewährungsstrafe davongekommen ist. Sie können sehr schnell im Internet überprüfen, dass es oft so ist: Beim Stichwort Kindesmissbrauch und Bewährungsstrafe bekommt man Dutzende von Einträgen. Wenn man dagegen Steuerdelikt und Bewährung googelt, stößt man auf ein Urteil des Bundesgerichtshofs: Er empfiehlt, dass ab einer Million Euro Steuerhinterziehung keine Bewährungsstrafe mehr ausgesprochen wird.

Michael Tsokos: Bei mir hat Sebastian damit offene Türen eingerannt. Ich komme oft als Sachverständiger in Gerichtsverhandlungen zu Wort und weiß genau, was Täter ihren Opfern antun. Wenn ich dann später von sehr milden Urteilen höre, denke ich oft, dass das nicht sein kann ${ }^{9}$.

In diesem Interviewausschnitt wird ein klarer Bezug des Inhalts des Textes zu den Irritationen hergestellt, die in der wirklichen Welt erfahrbar werden. Das geführte Gespräch stellt einen Beweis für die Aktualität des behandelten Themas in der heutigen Gesellschaft dar.

Wenn aber von einem literarischen Text die Rede ist, dann geht es nach Gansels Meinung letztlich um zwei Fragen: Die erste Frage betrifft das Was der Geschichte, gemeint ist das Dargestellte selbst. Die zweite Frage zielt darauf, wie die Geschichte erzählt wird, es geht um die Art und Weise des Erzählens, insbesondere darum, wer erzählt und aus wessen Perspektive die Welt dargestellt wird. Auf der Ebene der ,histoire' oder ,story' sind es vor allem die präsentierten Handlungen der Figuren, die in den Fokus der Aufmerksamkeit geraten. Mit anderen Worten: Es sind

9. - 22. Oktober 2012 Interview mit Sebastian Fitzek und Michael Tsokos, „Wo ist hier bitte die Minzpaste? “ http://www.buchjournal.de/554398/ (letzter Zugriff 23.06.2015). 
bevorzugt die entworfenen Figuren, Ereignisse, Geschehnisse, Räume, die aus den bisherigen Konventionen der Kriminalliteratur herausfallen und Irritation erzeugen.

Tsokos' und Fitzeks Abgeschnitten realisiert diese Irritation bzw. Störung über die Verbindung von Figur und Handlung. Offensichtlich ist aber auch, dass die Autoren die kalkulierte Aufstörung durch zu Anfang gesetzte Paratexte absichern.

Diese zwei Zitate aus führenden deutschen Tageszeitungen belegen den starken Realitätsbezug und wirken auf den Leser verstörend. Diese Zusammenführung von zwei sehr unterschiedlichen Verbrechen und den für das Vergehen errechneten Strafen gibt dem Leser einen ersten Denkanstoß, ob die Strafen gerecht waren, ob die Strafen gerecht verteilt worden sind. Gerecht nicht im Sinne des Rechtssystems, sondern der Moralvorstellung jedes einzelnen Lesers. Erst nach diesen Paratexten setzt der literarische Text ein. Mit der paratextuellen Einordnung wird die Aufmerksamkeit des Lesers auf den möglichen Realitätsbezug eines literarischen Textes gerichtet und seine aufstörenden Wirkungen provoziert. Tsokos' und Fitzeks Geschichte ist eine Variante der Verbrechen, die in der realen Welt begangen werden.

In einem weiteren Paratext zu Abgeschnitten, der die Vorgeschichte der Bekanntschaft Lindas, des Opfers des Stalkings, und Dannys, des Stalkers, erzählt, und auf der Internetseite www.ewig-mein.de in Form von einem Motion Comic veröffentlicht wurde, erfährt der Leser die Erweiterung des literarischen Textes durch das Einsetzen von Bildern und Musik. Dieser Einspieler wirkt fast wie eine Kampagne, deren Ziel es ist, junge Menschen über die Gefahren des Stalkings aufzuklären.

Ich bin zur Polizei gegangen. Sie müssen mir glauben, sie müssen es an meinem Gesicht sehen. Ich hatte mich getäuscht. Sie glauben etwa, dass mir etwas passiert war, aber niemand kaufte Danny Haag, dem Starautor einen wahnsinnigen Stalker ab. Niemand. Er war doch so charmant und verstand Frauen so gut. Ich hatte keine Beweise, seine Hände waren sauber [ ] Ich war nirgendwo sicher, nicht zu Hause, nicht bei der Polizei. Also zog ich um [ ] Richtig ausgepackt habe ich nie, als ob ich wüsste, dass er mich hier auch finden würde ${ }^{10}$.

Schon zu Anfang des Textes wird das Problem der Unglaubwürdigkeit der Opfer im Rechtssystem, das zwar als fiktives Organ im Text figuriert, jedoch Ähnlichkeiten mit dem wirklichen, realem System aufweist, eruiert.

[Danny] bereitete [es] anscheinend keine Probleme, wochenlang abzuwarten, bevor er wieder zuschlug, weshalb die Polizei sich nicht

10. - http://ewig-mein.de/. 
veranlasst gesehen hatte, gezielt gegen Danny vorzugehen. Nach Meinung der Behörden sprachen die für einen Stalker untypischen langen Intervalle, in denen Linda in Ruhe gelassen worden war, gegen einen einzelnen Täter. Viel wahrscheinlicher sei es, dass Linda einfach nur Pech gehabt hatte und zufällig von verschiedenen Männern belästigt worden war (,Von fanatischen Lesern Ihrer Comics vielleicht?“), und genau diese Einschätzung hatte Danny provozieren wollen. Zudem war er ein bekannter Autor, wohlhabend und gutaussehend, also einer, der ,jede kriegen kann", wie die Beamtin bei der Aufnahme der Anzeige angemerkt hatte, so als wäre Linda der Nachstellung Dannys gar nicht wert, über die sie sich hier beschwerte. Aber das hat Clemens [Bruder von Linda] ja gleich gesagt: Die Gesetze waren ein Witz, ihre Hüter ein Lacher. „Solche Sachen muss man selbst in die Hand nehmen (A, 26-27).

Wo verbirgt sich hier der aufstörende Charakter dieser Textstelle? Lindas Anliegen wird von der Behörde als ihre Einbildung gedeutet, da ihre Schilderungen der Situation nicht den Statistiken entsprechen, das Profil des Täters - bekannt, wohlhabend und gut aussehend - wird nicht als gefährlich erkannt und das Opfer - an anderer Stelle als hysterisch und gewalttätig beschrieben - wird als unglaubwürdig eingestuft. Linda erhält keine Hilfe seitens der Behörden, im Gegenteil, sie wird unseriös behandelt, beinahe ausgelacht, davon zeugen die Textstellen, in denen die Beamtin selber zum Wort kommt - im Text kursiv gekennzeichnet. Nach der Beschreibung von Lindas Versuch, eine Anzeige gegen Danny zu erstatten, kommt der Wechsel des point of view, eine Stimme spricht ein urteilendes Wort, das wie eine zusammenfassende Reflexion über das Gehörte wirkt: „Die Gesetze waren ein Witz, ihre Hüter ein Lacher" (A, 26). Danach werden die Worte Clemens' genau wiedergegeben: „Solche Sachen muss man selbst in die Hand nehmen“ (A, 27). Linda versteckt sich vor ihrem Peiniger auf der Insel Helgoland, jedoch enden ihre Bemühungen, Danny zu entkommen, mit einem Misserfolg, es kommt zu einem versuchten Mord. Danny wird versuchen, Linda in einem mit Wasser gefüllten Spülbecken zu ertränken. Dieser Strang des Romans zielt gegen die Vernachlässigung der Pflicht der Behörden, den Opfern zu helfen, auf ihre Klagen zu reagieren, ihre Anzeigen wahrzunehmen. Die Missachtung dieser Funktion führt zu traumatischen Erfahrungen der sich selbst überlassenen Opfer, zum Verbrechen, auch, wie im Text dargestellt, zur Selbstjustiz, als der einzigen Möglichkeit, sich von einem Täter zu befreien.

Gerade das Phänomen der Selbstjustiz gestaltet im Text das Bindeglied zwischen den beiden zuerst zweispurig geführten Erzählungen - der Stalking-Episode mit der Hauptfigur Linda im Zentrum und der Schnitzeljagd nach dem entführten Mädchen - in der auch Linda eine wichtige Rolle zufällt. In beiden Fällen wird das Versagen der 
Ordnungskräfte und der Justiz hervorgehoben. In beiden Fällen wird demonstriert, wie Opfer mit ihrem Problem alleine gelassen werden, wie sie selber zur Gewalt greifen, um sich selbst und andere potenzielle Opfer zu beschützen. In beiden Fällen wird der Prozess geschildert, wie aus Opfern durch Ohnmacht und Trauma Täter werden. Die so geführte Handlung und ihre Akteure führen zur Entstehung einer Aufstörung im Symbolsystem des Textes. Seine Funktion ist, bei dem Leser einen „Informationsverarbeitungsprozess“ in Gang zu setzen. Störungen irritieren die Toleranzgrenzen von Systemen, in diesem Fall treffen zwei Systeme aufeinander - das System Justiz und das System Literatur. Carsten Gansel meint: Störungen sind keineswegs nur als „Unfälle“ anzusehen, als etwas zu Verhinderndes, sondern sie sind ein wesentliches Mittel, um gesellschaftliche Wandlungsprozesse anzuregen. Weil Störungen eine „Denormalisierung“ (J. Link) bedeuten, provozieren sie ein beständiges Bestimmen der existierenden Normen und führen zu einer Überprüfung des bestehenden kollektiven Konsens. Über Störungen werden die Grenzen eines Systems markiert.

Dass die Autoren durch ihr Wirken die Grenzen des Systems Justiz angreifen wollen, wird stets an ihren Äußerungen sichtbar. In dem hier schon zitierten Interview sprechen sie offen von der direkten Verbindung der fiktiven, literarischen Welt und der realen, für Tsokos, der Arbeitswelt:

Buchjournal: Sie erzählen von einem Sexualstraftäter, der nach der Entlassung gleich wieder vergewaltigt. Was sollte man hier anders machen?

Michael Tsokos: Möglicherweise wäre es gut, wenn es mehr psychiatrische Gutachten gibt. Denn die Motivlage solcher Täter ist oft so komplex, dass ein einzelner Gutachter ihnen nicht gerecht werden kann.

Sebastian Fitzek: Mein Ansatz ist, dass wir von einem Täterstrafrecht zu einem Opferstrafrecht kommen sollten. Zurzeit wird die Schuld des Täters bemessen, und dann wird nach einiger Zeit untersucht, ob er auf dem Weg der Besserung ist. Das ist auch richtig so. Aber es müsste ebenfalls untersucht werden, wie lange das Opfer unter den Folgen der Straftat zu leiden hat, und da gibt es für mich eine einfache Faustformel: Solange das Opfer unter den Folgen der Straftat leidet, muss es auch Konsequenzen für den Täter haben. Zudem sollte es nicht so sein, dass wir zwar viel Geld für Täter ausgeben und für immer neue Gutachten, aber die oft schwer traumatisierten Opfer sich selbst überlassen bleiben und möglicherweise von Sozialhilfe leben müssen ${ }^{11}$.

Es sind Worte engagierter Autoren, die in der Öffentlichkeit ausgesprochen werden. Sie klagen das System Justiz an und deuten an, dass

11. - http://www.buchjournal.de/554398/. 
sie durch ihren gemeinsam geschriebenen Roman Missstände aufzeigen wollen. Wie wird das Vorhaben auf der Ebene des Textes realisiert?

Die Richterin hat geglaubt, man könne einen Kinderschänder resozialisieren. Glauben Sie das auch, Herr Professor? Ich sage Ihnen, was ich darüber denke, wie man mit verurteilten Verbrechern umgehen sollte. [...] Ich denke, jeder Täter sollte solange leiden, wie sein Opfer. Und wie seine Angehörigen. Was in meinem Fall bedeuten würde: Sadler muss bis zu seinem Tod die schlimmsten Quallen aushalten. Schwintowski.Lily. Rebecca. Sadler. Herzfeld begann das Ausmaß des Grauens zu begreifen. Menschen wie Sadler sind nicht therapierbar. Sobald sie die Gelegenheit bekommen, suchen sie sich das nächste Opfer. Kaum war das Monster aus dem Knast, schlug er wieder zu. Und diesmal griff es sich meine Rebecca. [...] Hier hat er sie zwei Tage vergewaltigt. (A, 291)

In dieser Episode spricht der betroffene Vater. Die Figurenrede wird wiedergegeben, der Erzähler unterbricht den Redefluss, um die Gedanken Herzfelds zu schildern. Herzfeld wird allmählich bewusst, welche Grausamkeiten den Eltern der Mädchen durch den Täter zugefügt worden sind. Die Figur Schwintowski ist unmittelbar in den Vorgang involviert, seine Tochter wurde misshandelt und beging Selbstmord, wie auch seine Frau, und jetzt steht er selbst kurz vor dem Suizid. Die Figur äußert Gedanken, die nicht mit dem System Justiz konform sind, die auch nicht mit dem bisher von Herzfeld vertretenen Standpunkt übereinstimmen. Schwintowski macht grade das System für den Tod seiner Tochter, seiner Frau und seinen eigenen verantwortlich. Er klagt in seiner Rede das ganze System an:

'Es geht um das gesamte System, das Opfer zu Tätern macht', erklärte Schwintowski. 'Um die Polizei, die viel zu überlastet ist, um bei jeder Vermisstenanzeige eine Suchaktion zu starten. Es geht um Gerichte, die Steuersünder härter bestrafen als Kinderschänder. Um Psychologen, die Freigang für Vergewaltiger empfehlen, sobald sie ein Trauma in ihrer Kindheit entdecken, während man mich für meine illegalen Casinos am liebsten in eine Einzelzelle sperren würde. Und natürlich geht es um einen rechtsmedizinischen Apparat als Teil eines sogenannten Rechtsstaats, der am Ende nur den Tätern nützt und die Opfer ein zweites Mal betraft' (A, 295).

In dieser Figurenrede wird das ganze System Justiz angeklagt. Es ist zu vermuten, dass hier der point of view stets gewechselt wird. Es werden allgemeingültige Äußerungen - z.B. zur Polizei, zur Strafordnung - mit ganz gezielten, auf die Figur bezogenen - ,illegale Casinos“ - zusammengeführt. Das thematische Überschreiten der erzählten Geschichte, Reflexionen über das Thema Strafe für begangene Verbrechen, explizite 
Benennung der Verstöße gegen die Moral einer Gesellschaft führen zu einer aufstörenden Wirkung des Textes im Handlungssystem.

Schwintowski ist der Meinung, dass die Richterin falsch geurteilt hatte, dass ihr keine glaubwürdigen Gutachten zur Verfügung standen, dass die Strafen für Gewalttäter und Sexualverbrecher zu niedrig angesetzt werden und dass Herzfelds Weigerung, das Gutachten zu fälschen, $\mathrm{zu}$ einer nicht angemessenen Strafe für den Vergewaltiger geführt hatte. Diese knappe Sequenz des Monologs Schwintowskis und die darauf einsetzende Handlung Herzfelds signalisieren, wie Störungen, die einmal in Gang gesetzt werden, zu Veränderungen führen können. Insofern können Schwintowskis Sätze Veränderungen in der Denkweise Herzfelds herbeiführen, also das System, dessen Teil er als Rechtsmediziner ist, von innen verändern. Denn nach Luhmann zeigen die Systeme einen Evolutionscharakter und sobald sie die Notwendigkeit der Veränderung anerkennen, sind sie imstande, die Veränderungen in ihre Strukturen einzubauen. Weiterhin lässt sich feststellen, dass Störungen die Toleranzgrenzen von Systemen irritieren und sie gegebenenfalls verändern können. Wie sich diese Störung auf Herzfeld ausgewirkt hatte, zeigt eine weitere Textstelle:

Herzfeld hätte mit jeder Sekunde schwächer werden müssen, angesichts dessen, wie viel Blut er verlor [...], aber der Zorn belebte ihn. 'Ich bring dich um!', keuchte er und trat dem Vergewaltiger in die Magengrube. [...] Zu viele Stimmen schrien in seinem Inneren durcheinander. Er hörte Martinek, der ihm vorwarf, Lily ein zweites Mal getötet zu haben, und dafür betete, Paul [Herzfeld] könne eines Tages nachvollziehen, was es bedeutet, seine Tochter zu verlieren. 'Oh ja, Sven. Du hast es mir gezeigt.' Herzfeld trat Sadler in den Magen und hörte Rebecca ihren eigenen Namen brüllen, während sie aus Angst vor den Qualen in den Tod sprang. Er zog den Sadisten an den Haaren hoch [...] Sadlers gutturale Schreie schafften es nicht, die von Hannah [entführte Tochter von Herzfeld] zu übertönen: 'Ich hasse dich'. Er nahm das Schwein in den Schwitzkasten, setze das Messer an seiner Kehle an [...] Im Geiste sah er, wie Schwintowski ihm zunickte. Er hatte seine Lektion gelernt. Manchmal muss man die Vorschriften brechen, um das Richtige zu tun. [...] [Herzfeld] erinnerte sich an Rebeccas gequältes Gesicht. An das Blut zwischen ihren Beinen und den Ausdruck in ihren Augen, als sie erkannte, wer sie war und dass ihr nur eine einzige Möglichkeit bleib, die Sache zu beenden. So wie er hier, heute und jetzt. Und mit dem Widerhall der toten Kinderstimmen in seinem Kopf schnitt er Sadler die Kehle durch (A, 376-378).

Diese aufeinanderfolgenden Textstellen signalisieren, dass Störungen, verstörende Informationen, traumatische Erlebnisse in der Kommunikation zu Veränderungen, ja vielleicht auch $\mathrm{zu}$ Reparaturhandlungen führen und auf diese Weise eine Kommunikation 
in Gang setzen. Insofern hat Schwintowskis Auffassung des Geschehenen eine verändernde, abgesehen von moralischen Vorbehalten, auch eine konstruktive Funktion: Der Vergewaltiger und Mörder wird durch Herzfelds Handeln nie mehr die Gelegenheit haben, weiteren Mädchen und Familien Leid zuzufügen. Wie Schwintowski in seinem Monolog dargestellt hatte, hat das System Justiz keine Maßnahmen gegen Verbrecher dieser Art entwickeln können. Herzfelds Vorstellung von Recht und Gerechtigkeit wurde durch die erlebten Vorfälle so stark irritiert, dass ein Lernprozess (genauso im Text formuliert - „Er hatte seine Lektion gelernt") aktiviert wurde, der zu gravierenden Veränderungen von Herzfelds Verständnis führte.

Tsokos und Fitzek zeigen, wie es zu einer Destabilisierung des Systems kommt: Herzfeld ist der Überzeugung, dass das System, wie es sich darstellt, unwirksam ist und zur Vermehrung und nicht zur Minderung der begangenen Strafen führt. Deswegen verändert er sein Handeln und bei voller Geisteskraft verübt er den Mord an dem mehrfachen Vergewaltiger.

\section{Funktion der Gewaltdarstellung}

Ein Gummischwamm, so groß wie ein Golfball, steckte in ihrem Mund und drückte permanent auf die pochende Wunde im Zahnfleisch. Doch dieser Schmerz war eine willkommene Ablenkung. Ihr Vergewaltiger hatte eine neue Körperöffnung in ihrem Unterleib gefunden und schien sie zerreißen zu wollen. Sie schrie zehn Minuten, nur durch erstickungsartige Hustenanfälle unterbrochen, aber wegen des Beißballs drang kaum mehr als ein dumpfes Stöhnen hervor (A, 76).

Die hier präsentierte Stelle, die nur als Beispiel für die Vergewaltigungs- und Folterszenen in dem Roman steht, erfüllt eine bestimmte Rolle in dem Text. Es geht weniger um Inszenierungen von Gewaltmöglichkeiten, als um eine realistische Schilderung dessen, was Menschen anderen Menschen antun können. Solche Szenen bergen ein hohes Irriationspotenzial, das noch durch die Information gesteigert wird, dass der Verursacher des Leides bereits vor Gericht stand und für dieselbe Tat zu einer milden Strafe verurteilt wurde und wegen guten Betragens die Anstalt vorzeitig verlassen durfte. Diese und alle weiteren Gewaltszenen dieses Romans sollen erschüttern, irritieren und aufstören, sollen auf gravierende Fehler in der psychologischen und psychiatrischen Einschätzung der Gewalttäter aufmerksam machen, rufen nach einer Verbesserung der Prozedur der Begutachtung von Sexualtätern. Denn die Funktion der Literatur besteht darin, die Wirklichkeit zu beobachten und über die Präsentation von Geschichten auch mit so drastischen Stoffen und Themen eine Kommunikation über diese Welt 
zu ermöglichen. Die etablierten Normen können im literarischen Text zur Diskussion gestellt werden und dürfen durchbrochen werden. Der ständige Wechsel des point of view führt zur Entstehung von Empathie, zur Perspektivenübernahme seitens der Leser. Erst die Fähigkeit des Menschen, sich in den geistigen Zustand von anderen Personen hineinzuversetzen, führt zur Kommunikation mit einem literarischen Text, die Aufstörung geltender Konventionen bewirken kann. Fitzek konstatiert: „Die Gespräche mit Michael (Tsokos, Rechtsmediziner) haben mir gezeigt, dass das, was wir da beschreiben - so drastisch es auch ist -, immer noch weniger schlimm ist als oft die Realität ${ }^{12 . “}$

12. - http://www.buchjournal.de/554398/. 
